\title{
Adolescence at Risk: An Overview
}

\author{
Shaik Ali*
}

\begin{abstract}
Today, 1.2 billion adolescents stand at the crossroads between childhood and the adult world. Around 243 million of them live in India. About one-quarter of India's population are adolescents.(UNICEF). As they stand at these crossroads, so do societies at large - the crossroads between losing out on the potential of a generation or nurturing them to transform society. Adolescence is considered as a period of transition from childhood to adulthood. It is characterized by rapid physical growth, significant physical, emotional, psychological and spiritual changes. Adolescents - young people between the ages of 10 and 19 years are often thought of as a healthy group. Nevertheless, many adolescents do die prematurely due to accidents, suicide, violence, pregnancy related complications and other illnesses that are either preventable or treatable. Many more suffer chronic ill-health and disability. In addition, many serious diseases in adulthood have their roots in adolescence. For example, tobacco use, sexually transmitted infections including HIV, poor eating and exercise habits, lead to illness or premature death later in life. Like adults, adolescents can experience emotions, thoughts, and behaviors that are distressing, disruptive, and disabling. Because many of these problems are precursors to much more disabling disorders during later life, mental and behavioral problems in childhood and adolescence represent a very high cost to society in both human and financial terms. The problems of adolescents are multi- dimensional in nature and require holistic approach. This paper aims to reveal the various problems of the adolescence in India. It also makes an attempt to locate the significance of measures from a strategic perspective. The present study is based upon secondary sources.
\end{abstract}

Key words: Development, Health, Policy, Substance abuse, and Violence.

\section{INTRODUCTION:}

Adolescence is the second decade of life. It is a period of great physical and psychological change. It also brings changes in social interactions and relationships. It is a time of opportunity, but also of risk. Adolescence is the window of opportunity to set the stage for a healthy and productive adulthood and to reduce the likelihood of health problems in the years to come. Yet it can entail risk, as a period when health problems that have serious immediate consequences can occur or when problem behaviors that have serious adverse effects on health in the future can be initiated. There are sound public health, economic and human rights reasons for investing in the health and development of adolescents. Greater investment in adolescent health would help prevent the estimated 1.4million deaths that occur each year among 10-19 year olds due to road traffic accidents, complications during pregnancy and child birth, suicide, violence, and HIV/AIDS. It would also improve the health and well-being of many millions of adolescents who

*Lecturer in Sociology, Government Pre-University College, Kavital. Tq: Manvi. Dt:Raichur. Karnataka 
experience health problems such as depression or anemia; and promote the adoption of behaviors that help prevent health problems later in life, such as cardiovascular diseases and lung cancer resulting from physical inactivity and tobacco use initiated during adolescence. Finally, investing in adolescent health can prevent problems in the next generation, such as prematurity and low-birth weight in infants born to very young mothers. There is growing recognition of the economic benefits of investing in the healthy development of adolescents, and the economic costs of not doing so. In 2002, the UN General Assembly Special Session on Children recognized the need for the "development and implementation of national health policies and programmers for adolescents, including goals and indicators, to promote their physical and mental health". Almost all Member States are signatories to the UN Convention on the Rights of the Child, which clearly states that adolescents have the right to obtain the health information and services they need to survive, grow and develop to their full potential.

Adolescents - young people between the ages of 10 and 19 years - are often thought of as a healthy group. Nevertheless, many adolescents do die prematurely due to accidents, suicide, violence, pregnancy related complications and other illnesses that are either preventable or treatable. Many more suffer chronic ill-health and disability. In addition, many serious diseases in adulthood have their roots in adolescence. For example, tobacco use, sexually transmitted infections including HIV, poor eating and exercise habits, lead to illness or premature death later in life. Like adults, adolescents can experience emotions, thoughts, and behaviors that are distressing, disruptive, and disabling. Because many of these problems are precursors to much more disabling disorders during later life, mental and behavioral problems in childhood and adolescence represent a very high cost to society in both human and financial terms. Estimates vary, but globally it seems that 15 to $20 \%$ of all children/adolescents have one or more mental or behavioral disorders, and some studies have shown prevalence even greater than $20 \%$. When young people have mental problems, they are at higher risk for abuse and neglect, suicide, substance use, school failure, violence and criminal activities, mental illness in adulthood, and health-jeopardizing impulsive behaviors. The present paper is based upon secondary sources.

\section{Overview:}

Caught in the web of transition from childhood to adulthood, the adolescents and youth between 10 and 24 years of age are most acutely affected by such unprecedented, and often unmanageable, changes. The swiftly-changing global conditions are placing a great strain on the young people, modifying their behavior and relationships and exacerbating their health problems. In the late ' $80 \mathrm{~s}$ that the world community formally recognized how seriously the health of young people impacts on the health and development future generations. With the further realization that the current and future health of young people depends very much on their own actions, choices and behaviors, the World Health Assembly passed a special resolution in May 1989 to highlight these issues. This resolution urged Member States to give acceptable programmers and services to meet these needs. It also emphasized the role of families and communities, and most importantly, the young themselves. Thereafter, the International Conference on Population and Development (ICPD) in Cairo in 1994 also emphasized the special needs of adolescents and youth. Since then there have been some activities carried out by nongovernmental organizations relating to a few aspects of adolescent health. But countries and communities are, by and large, neglecting this critical age group. There are still virtually no special programmers or services to prepare this vulnerability for a healthy adulthood. 
In 2003, the Committee of the Convention on the Rights of the Child (CRC) issued a General Comment in which the special health and development needs and rights of adolescents and young people were recognized. These are further supported by the Convention on the Elimination of Discrimination Against Women (CEDAW) and the Right to Health. However, prevention strategies and prompt interventions can reduce morbidity and mortality, and allow children and adolescents to develop into responsible, contributing adults. In today's fast changing world, adolescents - people between the ages of 10 and 19 years - face a range of health and social challenges. Many adolescents make the transition to adulthood in good health while some others do not. In addition, many serious diseases in adulthood have their roots in behaviors initiated during adolescence. Public health response in needed to address their specific health needs to ensure that they realize their fullest potential and are able to contribute to national development.

The World Health Organization has identified the following.

- Most young people are healthy. However, More than 2.6 million young people aged 10 to 24 die each year, mostly due to preventable causes.

- About 16 million girls aged 15 to 19 give birth every year. Young people, 15 to 24 years old, accounted for $40 \%$ of all new HIV infections among adults in 2009. An estimated 150 million young people use tobacco. Approximately 430 young people aged 10 to 24 die every day through interpersonal violence. Road traffic injuries cause an estimated 700 young people to die every day (WHO).

- A much greater number of young people suffer from illnesses which hinder their ability to grow and develop to their full potential.

\section{Health Issues affecting young people:}

The World Health Organization (WHO) has identified some of the main health issues affecting young people and is described below:

\section{Early pregnancy and childbirth:}

About 16 million girls aged 15 to 19 years give birth every year - roughly $11 \%$ of all births worldwide. The vast majority of adolescents' births occur in developing countries. The risk of dying from pregnancyrelated causes is much higher for adolescents than for older women. The younger the adolescent, the greater the risk. Unintended pregnancy (both unplanned and unwanted) among adolescents is a common public health problem worldwide. Repeat pregnancies in this group also occur frequently and are related to increased risks of adverse outcomes for adolescent mothers and their babies. Pregnancy and childbirthrelated deaths are the number one killers of 15-19-year-old girls worldwide, with nearly 70000 annual deaths. At least 2 million more young women are left with a chronic illness or disability, which may bring them life-long suffering, shame, or abandonment. Physically immature and often with few resources, the youngest first-time mothers are most at risk. Moreover, each year 2.2-4 million adolescents resort to unsafe abortion. Ninety-nine per cent of maternal deaths occur in the developing world, most of which (an overwhelming $74 \%$ ) are preventable. 
The International Journal of Indian Psychology: Volume: 01 | Issue: 03 | ISSN 2348-5396

\section{HIV/AIDS}

Fifteen to 24 year olds accounted for an estimated $40 \%$ of all new HIV infections among adults worldwide in 2009. Every day, 2400 more young people get infected and globally there are more than 5 million young people living with HIV/AIDS. Young people need to know how to protect themselves and have the means to do so. This includes condoms to prevent sexual transmission of the virus and clean needles and syringes for those who inject drugs. Currently, only $36 \%$ of young men and $24 \%$ of young women have the comprehensive and correct knowledge they need to protect themselves from acquiring the virus.

\section{Malnutrition}

Many boys and girls in developing countries enter adolescence undernourished, making them more vulnerable to disease and early death. Conversely, overweight and obesity (another form of malnutrition with serious health consequences and important longer term financial implications for health systems) are increasing among young people in both low- and high-income countries.

\section{Mental health}

In any given year, about $20 \%$ of adolescents will experience a mental health problem, most commonly depression or anxiety. The risk is increased by experiences of violence, humiliation, devaluation and poverty, and suicide is one of the leading causes of death in young people.

\section{Tobacco use}

The vast majority of tobacco users worldwide began when they were adolescents. Today an estimated 150 million young people use tobacco. This number is increasing globally, particularly among young women. Half of those users will die prematurely as a result of tobacco use.

\section{Harmful use of alcohol}

Harmful drinking among young people is an increasing concern in many countries. Alcohol use starts at a young age: $14 \%$ of adolescent girls and $18 \%$ of boys aged 13-15 years in low- and middle-income countries are reported to use alcohol. It reduces self-control and increases risky behaviours. It is a primary cause of injuries (including those due to road traffic accidents), violence (especially domestic violence) and premature deaths.

\section{Violence}

Violence is one of the leading causes of death among young people, particularly males: an estimated 430 young people aged 10 to 24 years die every day through interpersonal violence. For each death, an estimated 20 to 40 youths require hospital treatment for a violence-related injury.

\section{Injuries}

Unintentional injuries are a leading cause of death and disability among young people. Road traffic injuries take the lives of a staggering 700 young people every day. 


\section{THE INDIAN SCENARIO:}

A large number of adolescents in India are out of school, malnourished, get married early, working in vulnerable situations, and are sexually active. They are exposed to tobacco or alcohol abuse. In India, Jobs are shrinking at an alarming rate. Privatization and globalization have further aggravated the problem instead of generating employment; they have rendered millions of hands idle. It is caused by the rapid growth of population, the slow economic growth and the defective education system. Unemployment people easily become criminals. They disturb internal peace in the country and tempt aggressive countries to invade it. It is caused by the rapid growth of population, the slow economic growth and the defective education system. Unemployment people easily become criminals. They disturb internal peace in the country and tempt aggressive countries to invade it.

These various factors have made adolescence to be scared of. India has huge population which has triggered number of problems arising out of it. The population, more particularly adolescence has been diminished due to several reasons. They are sometime go into the hands of organized criminals or suffered from disorganized family rooting into the unwanted situations. For instance, girls are made to jump into the prostitution. It affects their physical and mental health. Many adolescent girls become brides, get pregnant, and have children before they are physically, emotionally, and socially mature enough to be mothers. Married or unmarried, adolescent girls become pregnant for different reasons. For some, pregnancy is accidental and the results of experimenting with sexuality or of lack of knowledge about how to prevent conception. Others seek pregnancy and motherhood to achieve adult status or fill an emotional void. But most adolescent pregnancies have little to do with choice or mistake. Globally, the majority of adolescent girls who become pregnant are married and pressured to have a child. For others, pregnancy often results from abusive, forced, or coerced sex (WHO). The situation in India is not out of it. The problem of child labor is also mounting at the alarming rate which affects the physical, emotional and mental health of the children and adolescents.

\section{STRATEGIC PERSPECTIVES:}

Many technical and political agencies at the global, regional and national levels have been implementing a variety of interventions with diverse approaches to address a wide range of factors related to unintended pregnancies among adolescents. The goals of these agencies have included, among others: helping adolescents to change psychosocial risk and protective factors involving sexuality; increasing teens' knowledge about risks and consistent and safe use of contraceptives; and skills training to support their social inclusion and personal development.

At the present time, stakeholders ranging from teens 'parents, health-care providers, teachers, and policymakers need to understand better how to set up programmes that can be practical, evidence-based, culturally appropriate, acceptable for adolescents, and that can guarantee good results in terms of the goals to be achieved to the satisfaction of all those involved, principally adolescents. The Strategic perspectives in the context of India are as follows:

- The programmes and policies for adolescents should focus on their constructive development. 
The International Journal of Indian Psychology: Volume: 01 | Issue: 03 | ISSN 2348-5396

- Counseling in psychological, academic performance, health areas, and career guidance.

- Provision of economic and social opportunities to involve all of them in some constructive activities at the college and community level with less competition and more with a sense of cooperation.

- Youth clubs should be established in the community as platforms to exhibit their inner qualities.

- Awareness programmes should be conducted about the impact of bad habits.

\section{CONCLUSION:}

There is great need to promote adolescent development to achieve the progress of the nation. The adolescents should be encouraged to participate in educational and economically viable activities which will enable them to actively involved in the process of national development. There is a need for preventive and curative mental health care for adolescents and support appropriate community action. The programmes for adolescents should focus on the development of inter-personal communication skills, counseling in psychological, academic and health areas, career guidance and provision of economic and social opportunities to involve all of them in some constructive activities at the school and community level with less competition and more with a sense of co-operation. This can be achieved by Competency Building as a part of redefining measures from a strategic perspective.

\section{REFERENCES:}

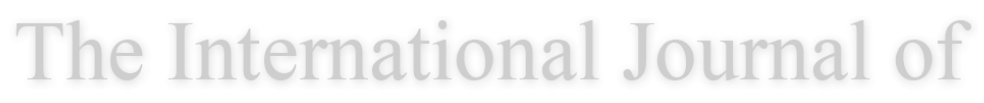

1. C. Eugene Walker; Michael C. Roberts(2001), 'Handbook of Clinical Child Psychology'. John Wiley \& Sons. New York.

2. Erik H. Erikson,(1994), 'Identity: Youth and Crisis'.W. W. Norton \& Company. New York.

3. http://www.who.int/topics/adolescent_health/en/. Retrieved 12.05.2014.

4. Inge Seiffge-Krenke Universitat Mainz,(1998),'Adolescents Health: A Developmental Perspective'. Lawrence Erlbaum Associates publishers,New Jersey London.

5. R Paruthi, PK Dutta (2002), 'Reproductive and child health programme', Indian Public Health Journal, Volume 46 | Issue 3-Sep.

6. www.google.com. Retrieved 12.05.2014. 